\title{
Forschungsaktivitäten und Innovationen in der Haarpflege - zukünftige Perspektiven in der Haarforschung
}

\author{
Research Activities and Innovations in Hair Care - Future Outlooks in \\ Hair Research
}

\section{Zusammenfassung}

Die Aktivitäten im Bereich der Haarforschung konzentrieren sich im Wesentlichen auf vier Bereiche: I) Haarmorphogenese, II) Haarwachstum und Haarausfall, III) Haarform sowie IV) Haarfarbe und Ergrauen. Dabei versucht man, die den verschiedenen Phasen des Haarfollikelwachstums zugrunde liegenden und sie regulierenden molekularen Mechanismen zu identifizieren, um damit auch gezielt in diese Prozesse eingreifen zu können. Zwar versteht man mittlerweile zahlreiche der Signale, die in der primären Haarfollikelbildung eine Rolle spielen. Ob diese Kenntnisse dabei helfen werden, die Übergangsphase vom Telogen zum Anagen zu beeinflussen, ist bislang allerdings unklar. Ein weiterer Forschungsschwerpunkt ist die Lokalisierung von Stammzellen, die für die zyklische Regenerierung des Haarfollikels unverzichtbar sind.
Abstract

The activities in the segment of hair research essentially concentrate on four ranges: i) hair morphogenesis, ii) hair growth and hair loss, iii) hair shape as well as iv) hair colour and hair greying. While doing so, we try to identify the different regulating molecular mechanisms, which underlie the different phases of hair follicle growth for the purpose of focused interventions on these processes. Indeed, we understand until today numerous signals, which play a role in the primary hair follicle development. But how this knowledge will help us to influence the transformation period from the telogen to the anagen, is not yet clear. Another research priority lies in the localisation of the stem cells, which are essential for the cyclic regeneration of the hair follicle.

\section{Anatomie und Morphogenese des Haarfollikels}

Der Haarfollikel ist ein sehr komplexes Organ aus mehreren Kompartimenten, die sehr gut aufeinander abgestimmt interagieren. Er ist das einzige Organ des menschlichen Körpers, das sich beim Erwachsenen ständig erneuert. Mittlerweile wurden zahlreiche der in einem riesigen Netzwerk miteinander kommunizierenden Akteure der Follikelmorphogenese identifiziert. Ihre zeitliche und räumliche Koordination im Rahmen dieses Prozesses ist allerdings noch weitgehend unbekannt, sodass eine kontrollierte Beeinflussung dieses Vorganges noch in weiter Ferne liegt.

Im Rahmen der Embryogenese entwickelt sich der Follikel aus epithelialen und bindegewebigen Anteilen (Abb.1). Ungefähr in der zehnten Embryonalwoche entsteht der Haarfollikel als Epithelknospe. Die Haarpapille dagegen entwickelt sich unterhalb des Follikels als bindegewebige Verdichtung aus Mesenchymalzellen und Fibroblasten. Sie wird während des weiteren Wachstums des Epithelknospe von diesem umschlossen, sodass die Haarknospe entsteht. Anschließend differenzieren sich aus zwei Epithelwülsten Talgdrüse und Wulstregion; außerdem entwickelt sich der Musculus arrector pili.

Der Haarfollikel ist in vier konzentrische Kompartimente unterteilt, die sich wiederum in Subkompartimente untergliedern. Von außen nach innen findet man I) das dermale Kompartiment mit der Bindegewebshülle und der dermalen Papille, II) den äußeren Teil der Wurzelscheide (ORS), III) den inneren Teil der Wurzelscheide (IRS) und IV) den Schaft selbst. Von unten nach 


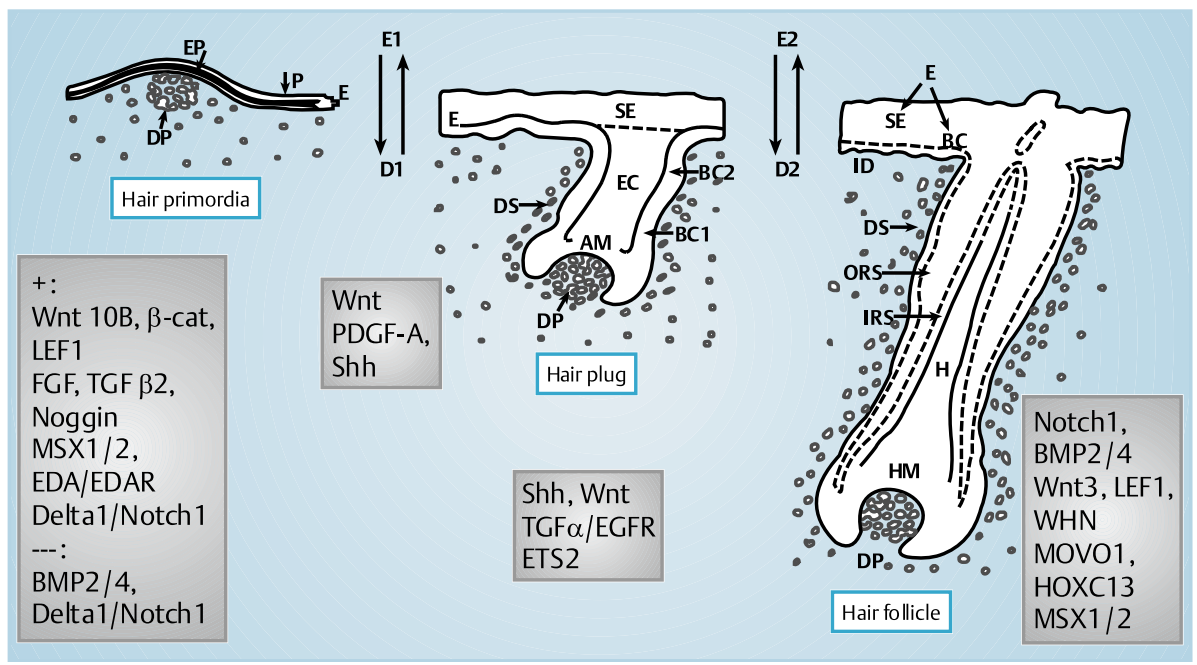

Abb. 1 Embryonale Entwicklung des Haarfollikels (Quelle: L'Oréal-Recherche).

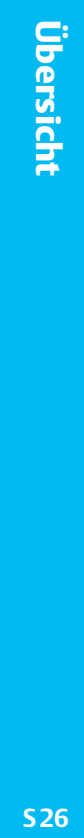

oben folgen nacheinander I) der Bulbus mit der proliferativen Matrix, II) der Isthmus mit dem Musculus arrector pili und der Talgdrüse sowie III) das Infundibulum, das sich von der Einmündung der Talgdrüse in den Follikelkanal bis zur Hautoberfläche erstreckt.

Umgrenzt wird der Follikel von der diskontinuierlich über das Organ verteilten Basalmembran. Auch hinsichtlich der weiteren Komponenten zeichnet sich der Follikel durch eine ausgeprägte Heterogenität aus. Eine Vielzahl von Substanzen wird nur in einzelnen Subkompartimenten, z. B. in Basalmembran, dermaler Papille oder bindegewebiger Scheide, exprimiert. So Laminin 5, ein Glykoprotein der Basallamina und Ligand des $\alpha 6 \beta 4$-Integrins, das nur entlang des Follikels, nicht aber rund um die Matrix exprimiert wird [3]. Auch die verschiedenen Keratine (K) werden im Haarfollikel mosaikartig synthetisiert [4]. Kann im oberen ORS die Expression von K5, K8, K14, K15, K17, K18, K19 und K20 sowie von Desmoglein und der Transglutaminasen 1 und 5 gemessen werden, so werden im unteren ORS nur K5, K8, K14, K17, K18 und K19 exprimiert. Dieses Expressionsmuster verdeutlicht die strikte Kontrolle des Differenzierungsprogramms in den einzelnen Follikelkompartimenten. Auch Haarfollikel und Epidermis werden sehr unterschiedlich reguliert: Im Gegensatz zu den zahlreichen im Follikel exprimierten Keratintypen werden in der Epidermis nur K1, K4, K10 und K14 synthetisiert.

Ein besonders komplexes Subkompartiment des Follikels ist die dreilagige IRS, die sich aus Kutikula, Huxleyscher Schicht und Henlescher Schicht zusammensetzt und in der ein zeitlich und räumlich strikt reguliertes Differenzierungsprogramm abläuft. So wird Trichohyalin nur zu Beginn des Differenzierungsprozesses in den mittleren Matrixzellen der Huxleyschen Schicht exprimiert. Erst später, wenn sich die Schichten der inneren Wurzelscheide zu differenzieren beginnen, setzt die Expression der Transglutaminase 1 ein. Die IRS ist von großer Bedeutung für die Aufrechterhaltung der Follikelintegrität: Sie wird benötigt, um den Differenzierungsprozess der ORS von dem des Haarschaftes zu separieren.
Auch der Haarschaft ist durch eine sehr differenzierte Keratinexpression charakterisiert. Insgesamt werden hier - ebenfalls unter sehr genauer zeitlicher und räumlicher Kontrolle - sieben saure sowie sechs unterschiedliche basische Keratine exprimiert. So wird das Keratin hHa5 ausschließlich in der keratogenen Zone synthetisiert, während die Expression von hHb2 auf die Kutikula beschränkt ist [5]. Zudem muss die Expression dieser Keratine auf die Synthese Keratin-assoziierter Proteine (KAP) abgestimmt werden, die Querverbindungen mit den Keratinen ausbilden. Bislang konnten 90 verschiedene KAP identifiziert werden, deren Rolle beim kontrollierten Aufbau des Haarschaftes bislang nicht geklärt ist. Bessere Kenntnisse hinsichtlich der funktionellen Bedeutung der Keratine und KAP könnten es jedoch ermöglichen, zukünftig die Charakteristika des Haarschaftes zu beeinflussen.

Am oberen Pol ist die dermale Papille, die Steuerungszentrale des Haarfollikels, von Haarmatrixzellen umgeben. Diese Zellen sind die am stärksten proliferierenden Zellen des menschlichen Organismus. Im Anschluss an die Zellteilung wandern sie den Bulbus hinauf und differenzieren sich zu den Zellen von Haarschaft bzw. IRS. Mittlerweile konnte unsere Arbeitsgruppe mehrere Mastergene identifizieren, die für die Regulation des Differenzierungsprogramms von Bedeutung sind. So ist beispielsweise Lef- $1 / \beta$ cat für die Differenzierung von Zellen im Haarschaft, GATA-3 für die Differenzierung in der IRS verantwortlich $[6,7]$.

\section{Haarwachstum und Haarausfall}

Das Wachstum des menschlichen Haares läuft in immer wiederkehrenden Zyklen ab: Auf die Anagenphase, in der das Haar wächst, folgt als Übergang die Rückbildung in der Katagenphase, der Zylkus schließt mit der Telogenphase (Ruhephase). Unsere Arbeitsgruppe hat in experimentellen Untersuchungen einzelne Haarfollikel über bis zu zehn Jahre beobachtet, um die Dauer dieser verschiedenen Phasen zu ermitteln. Eine Anagenphase erstreckt sich im Durchschnitt über drei Jahre, doch kann die Phasendauer eines Follikels von Zyklus zu Zyklus sehr stark variieren. Bislang ist noch unbekannt, wie die Phasendauer reguliert wird. Es ist uns also noch nicht möglich, die Anagenphase gezielt zu verlängern, um so den Haarausfall zu verhindern oder zumin- 


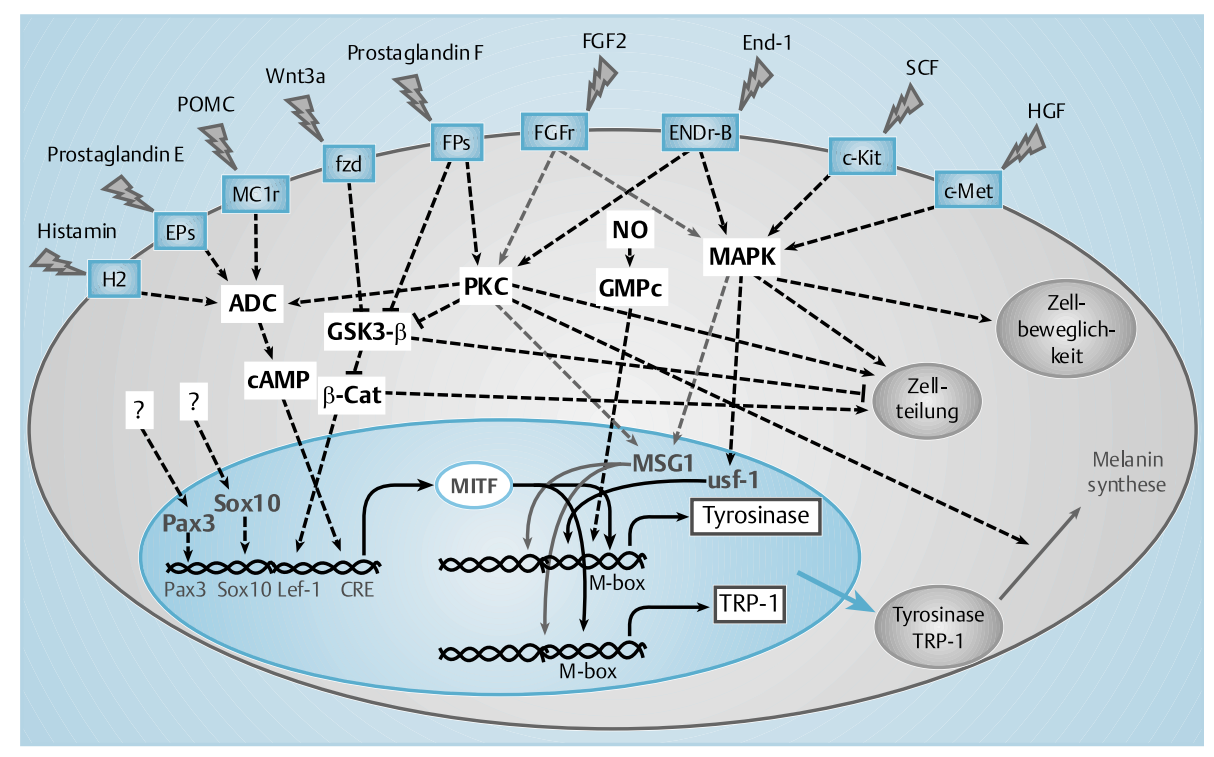

Abb. 2 Die Melanozytenaktivierung ist ein sehr komplex organisierter Vorgang des Haarfollikels (Quelle: L’Oréal-Recherche).

dest zu begrenzen. Klar ist heute jedoch, dass jeder Follikel die Phasenübergänge individuell und unabhängig von allen anderen Haarfollikeln über einen stochastischen Prozess kontrolliert.

Der Haarausfall wird über zahlreiche Faktoren reguliert. Eine wichtige Rolle spielen Hormone, Wachstumsfaktoren, Zytokine, Vaskularisierung und Innervation. Dabei können Follikel in unterschiedlichen Kopfhautregionen sehr unterschiedlich auf diese Faktoren reagieren, sodass sich ein typisches lokoregionales Muster des Haarausfalls ausbildet.

Nach Abschluss jedes Haarzyklus regeneriert sich der Follikel aus Stammzellen [8,9]. Mittlerweile konnten zwei Stammzellreservoire im distalen und proximalen ORS des Haarfollikels identifiziert werden. Welche Rolle diese beiden Reservoire spielen, ist allerdings derzeit wie so vieles andere bei der Regulation des Haarwachstums noch unbekannt.

\section{Form der Haare}

Die Form der Haare ist genetisch festgelegt. Programmiert wird die Ausbildung der Haarform von der Haarwurzel her, wie Studien mit in vitro kultiviertem Haar demonstrieren: Unabhängig von ihrer Umgebung, d.h. auch außerhalb der Kopfhaut, wachsen Haare in der genetisch vorgegebenen Form weiter. Lockiges Haar entsteht aufgrund eines asymmetrischen Differenzierungsprozesses: Sowohl die Zahl der sich teilenden Zellen als auch die Zusammensetzung der Kortikalzellen aus verschiedenen Keratinen und Matrixproteinen differiert zwischen konkaver und konvexer Seite des Haares [10]. Dagegen ist die Differenzierungsachse bei glattem Haar symmetrisch organisiert.

\section{Haarfarbe}

Die Pigmentierungseinheit des Haarfollikels befindet sich im oberen Teil der dermalen Papille (Bulbus) und besteht aus spezialisierten Melanozyten, die konstitutiv Melanin produzieren [11]. Das Pigment wird dann in Melanosomen gespeichert und in dieser Form an benachbarte Keratinozyten abgegeben. Wie der Melanosomentransport abläuft, ist bislang noch nicht geklärt. Die Melanozyten sind nur in der Anagenphase aktiv. Am Ende jedes Zyklus geht die Pigmentierungseinheit des Follikels unter und muss zu Beginn des nächsten Zyklus regeneriert werden.

Die Aktivierung der einzelnen Melanozyten zur Melanogenese ist sehr komplex organisiert. Eine Vielzahl von Hormonen, Wachstumsfaktoren und Mediatoren aktivieren über Rezeptoren auf der Zelloberfläche Signalkaskaden, die dann im Zellkern die Expression der für die Melaninproduktion verantwortlichen Enzyme einleiten (Abb. 2). Eine gezielte Manipulation dieser zahlreichen Signalwege und damit auch eine Beeinflussung der Haarfarbe erscheint derzeit noch unmöglich.

Im Haarfollikel existieren zwei unterschiedliche MelanozytenKompartimente: Während die Zellen der Pigmentierungseinheit aktiv Melanin synthetisieren, befinden sich die Melanozyten in der ORS im Ruhezustand. Sie stellen das für die Erneuerung der Pigmentierungseinheit am Ende jedes Haarzyklus verantwortliche Reservoir dar [12]. Die Mechanismen, die die MelanozytenRekrutierung und Aktivierung steuern, sind ebenso wie die Auslöser der Melanogenese noch unbekannt.

Auch in ergrauendem Haar synthetisieren die Melanozyten der Pigmentierungseinheit weiterhin Melanin. Das Ergrauen ist nicht durch eine verringerte Produktion von Melanin, den an der Melanogenese beteiligten Enzymen und einem reduzierten Melanosomen-Transfer bedingt, sondern lässt sich vielmehr auf eine Abnahme der Gesamtzahl an Melanozyten zurückführen. Mit zunehmendem Lebensalter sinkt sowohl die Zahl der Melanozyten in der Pigmentierungseinheit als auch die der ruhenden Zellen im Reservoir [13]. Dieser Rückgang betrifft allerdings nur die Melanozyten des Haarfollikels, nicht aber die von Epidermis und Infundibulum, deren Zahl konstant bleibt.

Zusammenfassend lässt sich festhalten, dass bisher etliche Prozesse, die Haarmorphogenese, Haarwachstum und Haarausfall, Haarform sowie Haarfarbe bestimmen, aufgeklärt werden konn- 
ten. Dennoch gibt es heute zu all diesen Vorgängen weiterhin mehr Fragen als Antworten. Erst die genauere Erforschung der molekularen Mechanismen, die in den verschiedenen Phasen des Haarzyklus eine Rolle spielen, wird eine gezielte Beeinflussung kosmetischer und medizinischer Haarprobleme erlauben.

\section{Literatur}

${ }^{1}$ Botchkarev VA, Paus R. Molecular biology of hair morphogenesis: development and cycling. J Exp Zoolog Part B Mol Dev Evol 2003; 298: $164-180$

2 Tobin DJ, Gunin A, Magerl M, Paus R. Plasticity and cytokinetic dynamics of the hair follicle mesenchyme during the hair growth cycle: implications for growth control and hair follicle transformations. J Investig Dermatol Symp Proc 2003; 8: 80-86

${ }^{3}$ Commo S, Bernard BA. The distribution of a2b1, a3b1 and a6b4 integrins identifies distinct subpopulations of basal keratinocytes in the outer root sheath of the human anagen hair follicle. Cell Mol Life Sci 1997; 53: 466-471

${ }^{4}$ Eichner R, Kahn M. Differential extraction of keratin subunits and filaments from normal human epidermis. J Cell Biol 1990; 110: 11491168
${ }^{5}$ Thibaut S, Collin C, Langbein L, Schweizer J, Gautier B, Bernard BA. Hair keratin pattern in human hair follicles grown in vitro. Exp Dermatol 2003; 12: $160-164$

${ }^{6}$ Botchkarev VA, Botchkareva NV, Roth W, Nakamura M, Chen LH, Herzog W, Lindner G, McMahon JA, Peters C, Lauster R, McMahon AP, Paus R. Noggin is a mesenchymally derived stimulator of hair-follicle induction. Nat Cell Biol 1999; 1: 158 - 164

${ }^{7}$ Kaufman CK, Zhou P, Pasolli HA, Rendl M, Bolotin D, Lim KC, Dai X, Alegre ML, Fuchs E. GATA-3: an unexpected regulator of cell lineage determination in skin. Genes Dev 2003; 17: 2108-2122

${ }^{8}$ Commo S, Gaillard O, Bernard BA. The human hair follicle contains two distinct K19 positive compartments in the outer root sheath: a unifying hypothesis for stem cell reservoir? Differentiation 2000; 66:

${ }^{9}$ Brouard M, Barrandon Y. Controlling skin morphogenesis: hope and despair. Curr Opin Biotechnol 2003; 14: 520-525

${ }^{10}$ Bernard BA. Hair shape of curly hair. J Am Acad Sci 2003; 48: S120S126

${ }^{11}$ Botchkareva NV, Botchkarev VA, Gilchrest BA. Fate of melanocytes during development of the hair follicle pigmentary unit. J Investig Dermatol Symp Proc 2003; 8: 76-79

${ }^{12}$ Commo S, Bernard BA. Melanocyte subpopulation turnover during the human hair cycle: an immunohistochemical study. Pigment Cell Res 2000; $13: 253-259$

${ }^{13}$ Commo S, Gaillard O, Bernard BA. Human hair greying is linked to a specific depletion of hair follicle melanocytes affecting both the bulb and the outer root sheath. Br J Dermatol 2004; 150: 435-443 